\title{
Transcatheter tricuspid valve replacement with a self-expanding bioprosthesis
}

Andrea Colli, MD, PhD, ${ }^{\mathrm{a}}$ Gino Gerosa, MD, ${ }^{\mathrm{a}}$ Krzysztof Bartus, MD, PhD, ${ }^{\mathrm{b}}$ and Giuseppe Tarantini, $\mathrm{MD}, \mathrm{PhD}^{\mathrm{a}}$

From the ${ }^{\text {a} D e p a r t m e n t ~ o f ~ C a r d i a c, ~ T h o r a c i c, ~ a n d ~ V a s c u l a r ~ S c i e n c e s, ~ U n i v e r s i t y ~ o f ~ P a d u a, ~ P a d u a, ~ I t a l y ; ~ a n d ~}$ ${ }^{\mathrm{b}}$ Department of Cardiovascular Surgery and Transplantology, Jagiellonian University, John Paul II Hospital, Kraków, Poland.

No funding was provided for this work.

Drs Colli and Tarantini contributed equally to this article.

Disclosures: Authors have nothing to disclose with regard to commercial support.

Received for publication Oct 29, 2017; revisions received Feb 24, 2018; accepted for publication March 24, 2018; available ahead of print April 27, 2018.

Address for reprints: Andrea Colli, MD, PhD, and Giuseppe Tarantini, MD, PhD, Department of Cardiology, Thoracic and Vascular Sciences, University of Padua, Via Giustiniani, 2, Padova 35128, Italy (E-mail: andrea.colli@unipd.it or colli.andrea.bcn@gmail.com or giuseppe.tarantini.1@unipd.it or giuseppe.tarantini. $1 @$ gmail.com).

J Thorac Cardiovasc Surg 2018;156:1064-6

$0022-5223 / \$ 36.00$

Copyright (C) 2018 by The American Association for Thoracic Surgery

https://doi.org/10.1016/j.jtcvs.2018.03.096

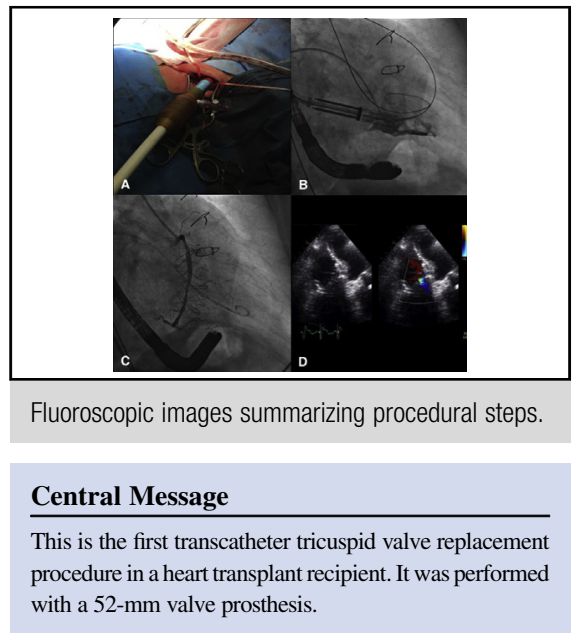

See Editorial Commentary page 1067.

$\checkmark$ Video clip is available online.

A 66-year-old male patient, 27 years after heart transplantation and with stage 3 chronic kidney disease, presented with severely symptomatic and medically refractory severe tricuspid valve (TV) regurgitation with ascites and spontaneous prolonged prothrombin time (euroSCORE, II 39.23\%; Video 1). MitraClip (Abbott Laboratories, Abbott Park, Ill) use was not considered feasible because of the presence of concomitant severe annular dilatation (43 $\mathrm{mm})$ and flailing of the posterior leaflet. The patient was accepted by the heart team for transcatheter TV replacement with the GATE bioprosthesis ${ }^{1}$ (NaviGate Cardiac Structures Inc, Lake Forest, Calif) after approval was received from national authorities for compassionate use. Cardiac computed tomographic scan was used to visualize the angle and proximity between the TV annulus and the right coronary artery, which was selected as a prosthesis deployment landmark. Cardiac computed tomographic scan (3mensio Structural Heart version 9.0 SP1; Pie Medical Imaging, Maastricht, The Netherlands) was also used to simulate access site route and of final appearance of the device once implanted (Figure 1). Because of the patient's critical clinical condition, a maximal dosage of inotropic support was administered, and femoral venoarterial extracorporeal membrane oxygenation circulatory backup support was planned to be available. With the patient under

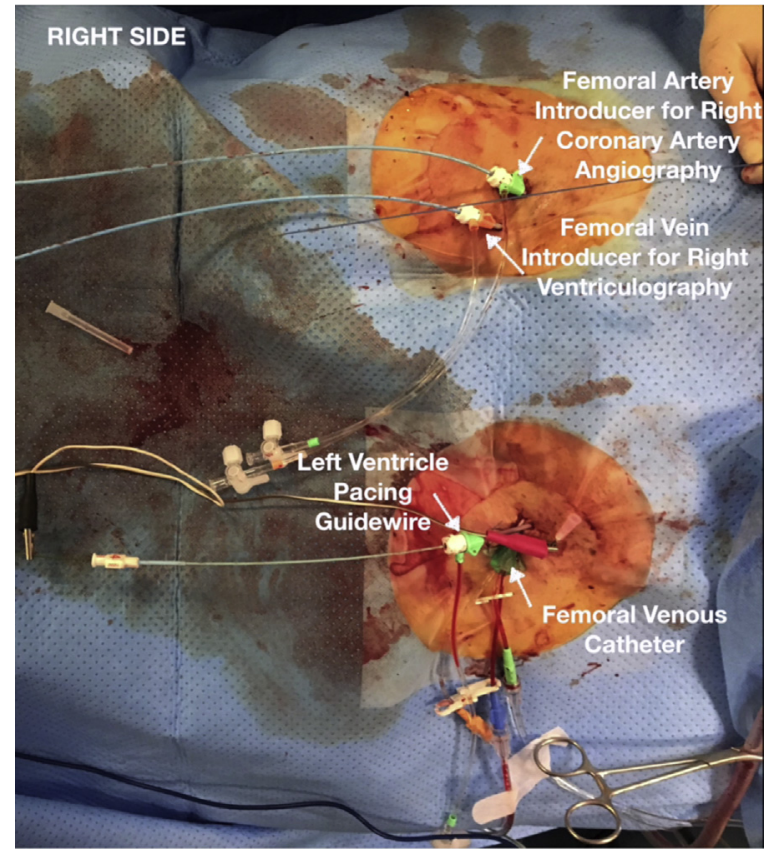

VIDEO 1. Transcatheter tricuspid valve replacement with GATE (NaviGate Cardiac Structures Inc, Lake Forest, Calif) bioprosthesis: preprocedural evaluation, procedural steps, and postprocedural assessment. Preop, Preoperative; $2 D$-TTE, 2-dimensional transthoracic echocardiography; $3 D-T T E$, 3-dimensional transthoracic echocardiography; $C T$, computed tomography; $R C A$, right coronary artery; $A V$, atrioventricular; $R A$, right atrium; $R V$, right ventricle; $P A$, pulmonary artery; postop, postoperative. Video available at: https://www.jtcvs.org/article/S0022-5223(18)30877-8/fulltext. 

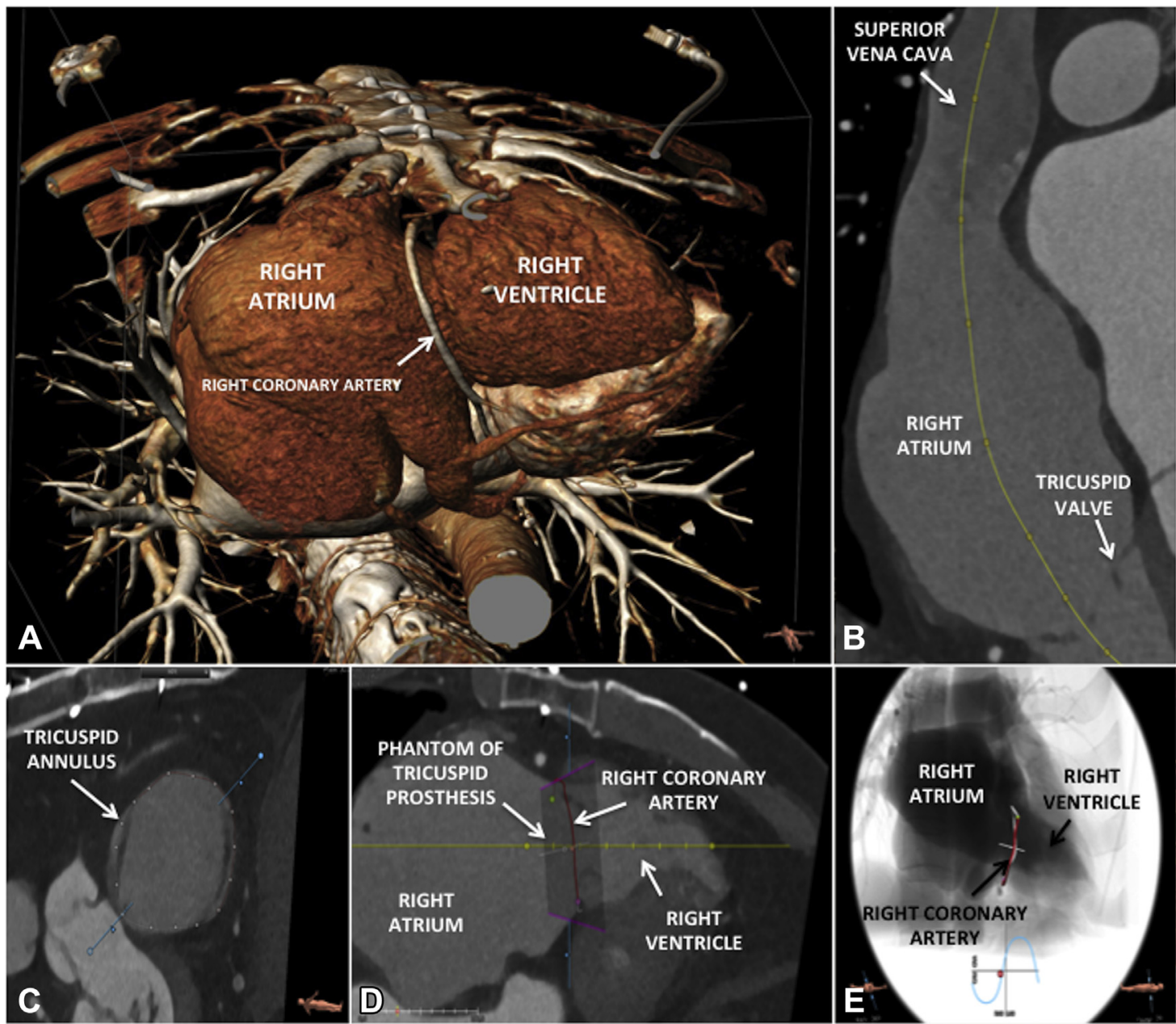

FIGURE 1. Preprocedural assessment on cardiac computed tomography. A, Volume-rendered cardiac computed tomography shows the right coronary artery course parallel to the right atrioventricular junction. B, Right-sided heart access route simulation. C, Three-dimensional tricuspid annulus segmentation. D, Long-axis view with simulated GATE conical device (38-52 mm diameters, height $21 \mathrm{~mm}$; NaviGate Cardiac Structures Inc, Lake Forest, Calif). E, Prediction of fluoroscopy angulation (3mensio Structural Heart version 9.0 SP1; Pie Medical Imaging, Maastricht, The Netherlands).

general anesthesia, a right surgical internal jugular vein approach was used to advance the $42 \mathrm{~F}$ introducer sheath (Figure 2). Two arterial accesses were required for right coronary artery angiography and temporary left ventricular pacing by means of a 0.035 -inch guidewire. An Amplatzer SuperStiff guidewire (Abbott) was placed into the right pulmonary artery. The delivery system $(36 \mathrm{~F})$ was advanced and steered across the TV to obtain perpendicularity; implantation height was judged with right coronary artery angiography. A 52-mm GATE prosthesis ${ }^{1}$ was deployed, resulting in a $2-\mathrm{mm} \mathrm{Hg}$ mean gradient and mild residual septal paravalvular leakage (Figure 3). The patient tolerated the procedure well, with fast and effective weaning from inotropic support, and was discharged home after 5 days.
After 64 days, atrioventricular block developed, which required leadless pacemaker implantation (Micra; Medtronic, Dublin, Ireland). At 6 months of follow-up, the patient is free of symptoms, and the valve was functioning well on most recent echocardiography. This is the first transcatheter TV replacement procedure performed in a heart transplant recipient, with the largest valve prosthesis ever implanted in humans.

\section{Reference}

1. Navia JL, Kapadia S, Elgharably H, Harb SC, Krishnaswamy A, Unai S, et al First-in-human implantations of the NaviGate bioprosthesis in a severely dilated tricuspid annulus and in a failed tricuspid annuloplasty ring. Circ Cardiovasc Interv. 2017;10 


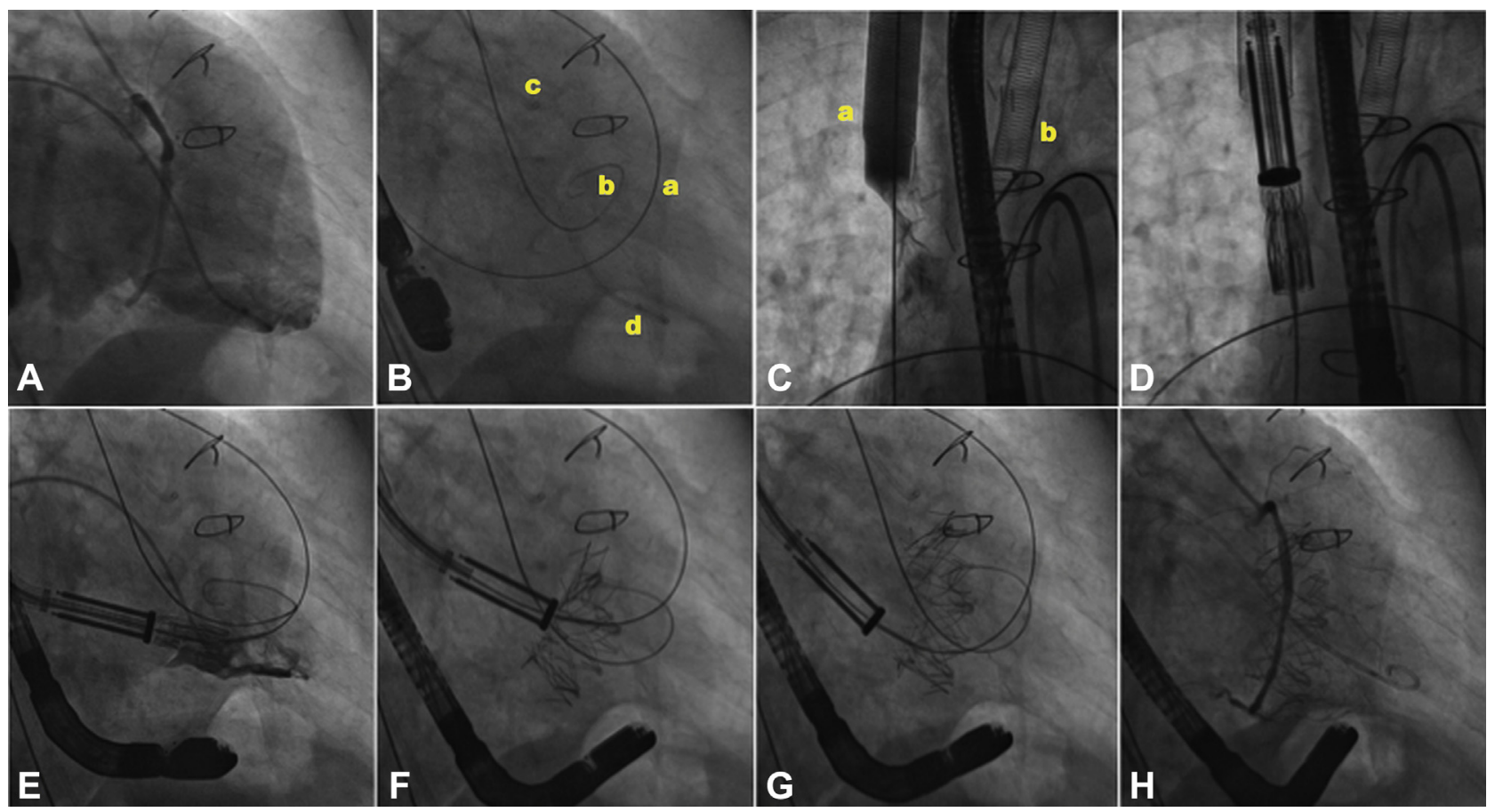

FIGURE 2. Fluoroscopic images summarizing procedural steps. A, Simultaneous right coronary artery angiography and right ventriculography for assessment of the deployment area. B, An Amplatzer SuperStiff guidewire (Abbott Laboratories, Abbott Park, Ill) was placed into the right pulmonary artery (a). A left ventricular guidewire was used as rescue pacemaker lead $(b)$. Right coronary artery $(c)$ and right ventriculography pigtail $(d)$ catheters can be seen. C, A $42 \mathrm{~F}$ introducer $(a)$ and a tracheal tube for lung ventilation $(b)$ were placed. D, The delivery system was advanced into the cavoatrial junction. E, The delivery system was positioned orthogonal to the tricuspid annulus. F, Partial GATE prosthesis (NaviGate Cardiac Structures Inc, Lake Forest, Calif) deployment. G, Complete deployment of the GATE prosthesis. H, Final appearance of the GATE prosthesis with respect to the right coronary artery.

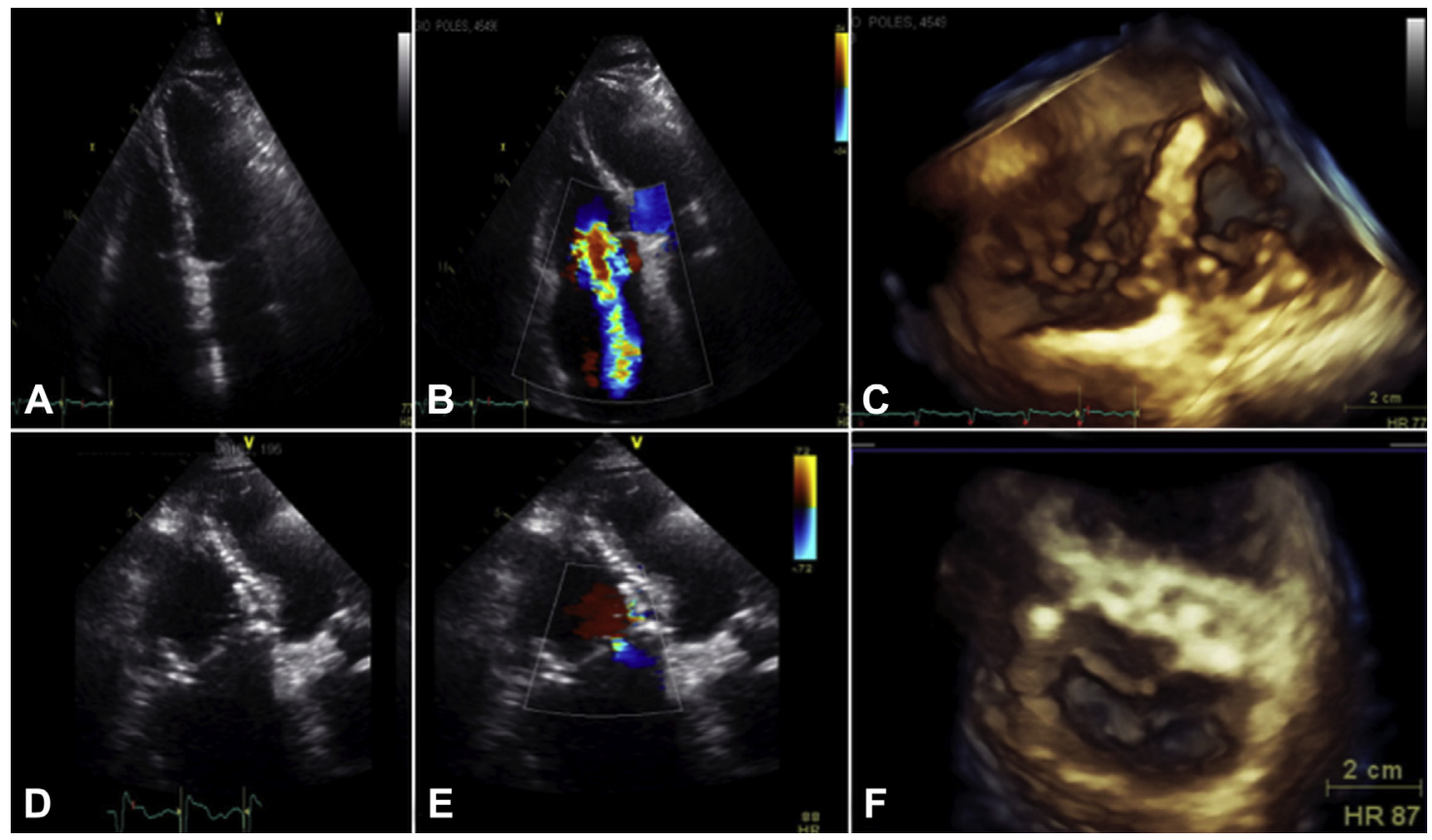

FIGURE 3. Echocardiographic Imaging before and after the procedure. A, Preoperative 2-dimensional transthoracic echocardiography shows severe annular dilatation. B, Color Doppler echocardiography shows severe tricuspid regurgitation. C, Preoperative 3-dimensional echocardiography of the tricuspid valve. D, Postoperative 2-dimensional transthoracic echocardiography shows the GATE prosthesis (NaviGate Cardiac Structures Inc, Lake Forest, Calif) in the tricuspid position. E, Color Doppler echocardiography shows the presence of mild septal paravalvular regurgitation. F, Postoperative 3-dimensional echocardiography visualization of the GATE prosthesis. 